\title{
Comment interpréter les indicateurs de mortalité intra-hospitalière?
}

\author{
Julien Sansonnens ${ }^{a}$, Luc Fornerod ${ }^{a}$, Arnaud Chiolero ${ }^{a, b}$ \\ a Observatoire valaisan de la santé, Sion; ${ }^{b}$ Institut universitaire de médecine sociale et préventive (IUMSP), CHUV, Lausanne
}

\begin{abstract}
La publication des indicateurs de mortalité est réalisée dans l'intérêt des patients et des hôpitaux, dans une visée d'amélioration de la qualité des soins. Les indicateurs sont des indices d'un problème potentiel, mais ils doivent être interprétés correctement. L'article propose une méthode pour faciliter l'interprétation et l'emploi de ces indicateurs.
\end{abstract}

\section{Contexte}

La qualité des soins devient une préoccupation majeure des patients, des médecins, des soignants, des assureurs et des personnes impliquées dans la gestion et la planification des systèmes de santé $[1,2]$. L'amélioration de la qualité des soins nécessite notamment un système de surveillance sanitaire permettant de suivre des indicateurs de qualité des soins. Un indicateur de qualité des soins est un élément mesurable quantitativement qui permet d'évaluer la qualité des soins ou de suivre le déroulement et les effets d'une intervention visant à améliorer la qualité des soins. Classiquement, on distingue des indicateurs de structure, de processus et de résultats [1]. Idéalement, un indicateur doit être valide, c'est-à-dire refléter la qualité des soins que l'on souhaite appréhender, reproductible, précis et sensible au changement [3,4].

La mortalité intra-hospitalière est fréquemment utilisée comme un indicateur de la qualité des soins [5, 6]. C'est un indicateur de résultat qui est analysé depuis 2008 par l'OFSP sur la base des données de la statistique médicale [7]. L'OFSP a choisi comme indicateurs le nombre de cas de 41 pathologies et procédures fréquentes et la mortalité associée. Depuis 2012, l'OFSP publie de manière transparente, c'est-à-dire avec la dénomination des établissements, des indicateurs de mortalité de tous les hôpitaux de soins somatiques aigus de Suisse [8]. La publication de ces données, reprises et commentées notamment par certains médias [9], est fortement débattue [10, 11]. La méthodologie utilisée par l'OFSP a été critiquée, notamment parce que les données proviennent du système de facturation des forfaits par cas ou parce que la méthode d'ajustement du risque de mortalité serait insuffisante. Une autre critique est la difficulté à établir un lien de causalité entre la pathologie ou la procédure et le décès, qui peut ne pas être en lien avec la pathologie ou la procédure.

Il est en effet reconnu que les indicateurs de mortalité intra-hospitalière ont des limites qui doivent être considérées lorsqu'ils sont analysés [14]. Néanmoins,

Un hôpital qui traite des cas moins sévères aura tendance à avoir une mortalité plus basse.

la publication des indicateurs de mortalité est réalisée dans l'intérêt des patients et des hôpitaux, dans une visée d'amélioration de la qualité des soins, et il est dès lors nécessaire que les indicateurs soient compris et intelligibles pour les utilisateurs. Nous proposons ci-dessous une méthode pour faciliter l'interprétation et l'emploi de ces indicateurs.

\section{Indice de mortalité standardisée}

L'OFSP publie les indices standardisés de mortalité [standardized mortality ratio (SMR)] pour certaines pathologies et procédures. Le SMR est le rapport entre le taux de mortalité observé dans l'hôpital (nombre de décès / nombre de cas) et le taux de mortalité attendu, calculé sur la base des taux observés en moyenne en Suisse et standardisés pour tenir compte de la distribution de l'âge et du sexe dans l'hôpital. Le SMR est donc:

- SMR = mortalité observée / mortalité attendue. Un SMR supérieur à 1 indique une mortalité observée supérieure à la mortalité attendue. L'interprétation des SMR est plus compliquée qu'il n'y parait et pose 
Tableau 1: Mortalité par une intervention et une pathologie, tirée de la publication "Indicateurs de qualité des hôpitaux suisses de soins aigus 2012» [7].

\begin{tabular}{lllllll}
\hline Intervention & $\begin{array}{l}\text { Taux } \\
\text { observé }\end{array}$ & $\begin{array}{l}\text { Taux } \\
\text { attendu }\end{array}$ & SMR & $\begin{array}{l}\text { Nombre } \\
\text { de cas }\end{array}$ & $\begin{array}{l}\text { Décès } \\
\text { observés }\end{array}$ & $\begin{array}{l}\text { Décès } \\
\text { attendus }\end{array}$ \\
\hline $\begin{array}{l}\text { 1. Première } \\
\text { implantation de } \\
\text { prothèse de hanche* }\end{array}$ & $1,0 \%$ & $0,2 \%$ & 5,4 & 105 & $\mathbf{1}$ & $\mathbf{0 , 2}$ \\
\hline $\begin{array}{l}\text { 2. Infarctus cérébral } \\
\text { (CIM-10 I63, âge }>19)\end{array}$ & $11,0 \%$ & $7,4 \%$ & 1,5 & 356 & 39 & $\mathbf{2 6}$ \\
\hline 3. Intervention fictive & $1,1 \%$ & $0,7 \%$ & 1,5 & 3560 & $\mathbf{3 9}$ & $\mathbf{2 6}$ \\
\hline
\end{tabular}

*EPT de hanche
Dans les deux cas, il y a une surmortalité dans cet hôpital, les taux de mortalité observés étant supérieurs aux taux attendus. En se basant uniquement sur le SMR, on pourrait en déduire que la mortalité après pose de prothèse de hanche est particulièrement inquiétante, alors que la mortalité après infarctus cérébral, plus proche de 1, l'est moins. Néanmoins, en tenant compte du nombre de décès observés et le nombre de décès attendus, en italique dans le tableau 1, l'interprétation est différente.

Ces nombres sont calculés en multipliant le taux observé, respectivement attendu, par le nombre de cas. Le nombre de décès attendus dans cet hôpital suite à une pose de prothèse de hanche est inférieur à 1 . Dès lors, un seul décès a conduit à un SMR important de 5,4. A l'opposé, l'écart entre le nombre de décès attendus (26) et le nombre de décès observés (39) est beaucoup plus important pour les infarctus cérébraux, indiquant une surmortalité importante. En tenant compte de l'écart entre le nombre observé et le nombre attendu, c'est la surmortalité pour les infarctus cérébraux qui est plus inquiétante, justifiant une évaluation plus poussée et, si nécessaire, la prise de mesures.

En pratique, nous proposons de calculer systématiquement, pour les interventions dont le SMR est supérieur à 1, le nombre de décès observés et attendus,

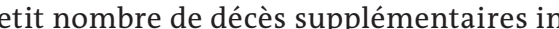
fortement le SMR, alors qu'ils sont le reflet d'une fluctuation aléatoire d'une année à une autre. A l'extrême, un seul décès supplémentaire peut amener à ce que le SMR devienne largement supérieur à 1 , ceci pouvant conduire à une interprétation trop alarmiste du résultat. L'interprétation des SMR est aussi plus aisée pour des pathologies ou procédures dont le volume est important, et ces indicateurs sont donc plus informatifs pour les hôpitaux qui ont le plus de cas [12].

\section{Aide à l'interprétation des indicateurs de mortalité}

Comme nous l'avons vu, la pertinence statistique du SMR est limitée lorsque le nombre de décès est faible (reflétant une faible mortalité ou un petit volume de cas). Dans ces cas, un SMR élevé ne suffit pas à identifier des situations potentiellement problématiques, et nous proposons de tenir compte de l'écart entre les nombres absolus de décès observés et de décès attendus. Prenons l'exemple d'une intervention (implantation de prothèse de hanche) et d'une pathologie (infarctus cérébral) effectuée ou traité en 2012, dans un hôpital non identifié, et issues de la publication «Indicateurs de qualité des hôpitaux suisses de soins aigus 2012» [7];

\section{Il faut mettre en relation le nombre de décès observés et attendus avec le nombre de diag- nostics ou d'interventions pratiquées.}

et d'utiliser cette information, conjointement au SMR, pour apprécier le caractère problématique ou non d'un résultat. L'utilisation de nombres entiers représentant des patients ou des décès est préférable en matière de communication du risque [13]. En effet, intuitivement, ces chiffres sont plus faciles à appréhender que des rapports tels que le SMR ou des proportions. Il est difficile de proposer un nombre de décès à partir duquel la différence entre décès attendus et décès observés peut être considérée comme inquiétante: il faut notamment tenir compte de la pathologie ou de la procédure considérée. Le jugement clinique est ici nécessaire.

Par rapport au seul SMR, l'écart entre le nombre de décès attendus et observés fournit une information supplémentaire, mais il faut la mettre en perspective avec le nombre total de cas. Sur le tableau 1, nous avons ajouté une ligne correspondant à une intervention fictive pour laquelle le SMR ainsi que les décès attendus et observés sont les mêmes que pour l'infarctus cérébral, ce qui pourrait laisser croire que la 


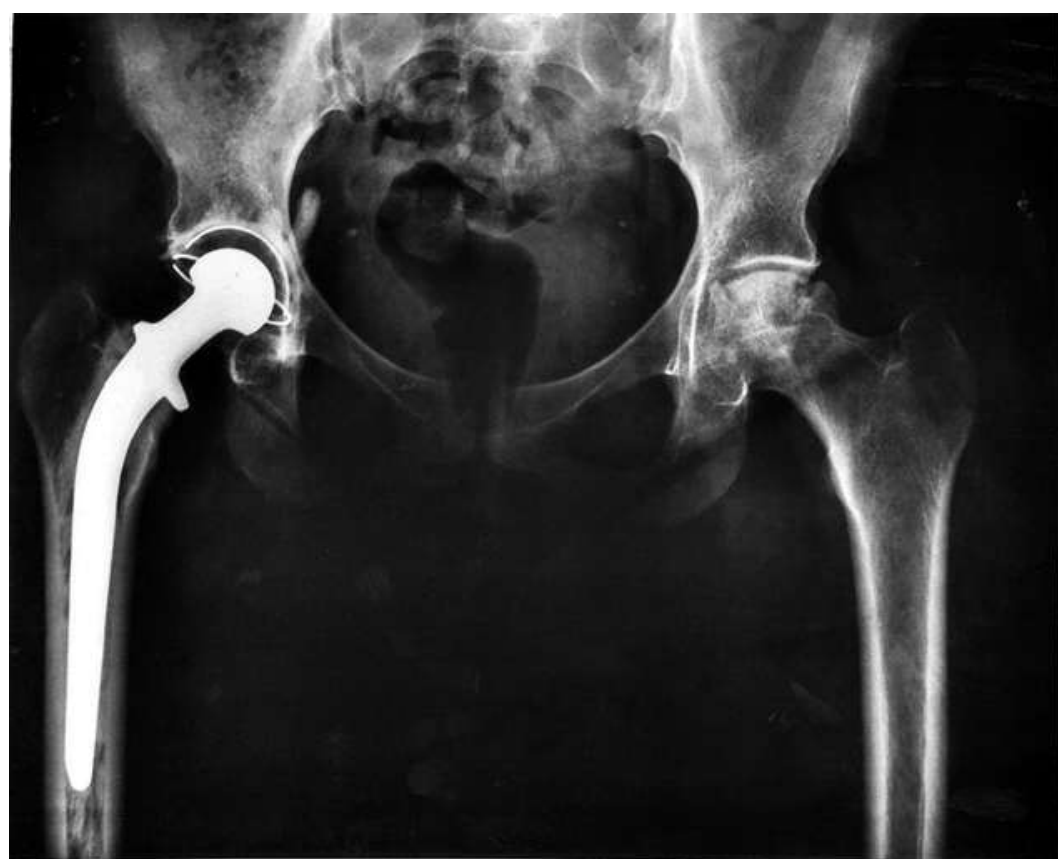

Une question pas si facile à répondre: est-ce que la mortalité suite à la pose d'une prothèse de hanche est particulièrement inquiétante?

mortalité est pareillement problématique dans les deux cas. Toutefois, ce même écart de 13 décès est plus préoccupant pour l'infarctus cérébral que pour cette intervention fictive, car cela correspond à une surmortalité, en pourcentage, beaucoup plus élevée pour l'infarctus cérébral. Pour apprécier le caractère problématique ou non d'un résultat, il faut mettre en relation le nombre de décès observés et attendus avec le nombre de diagnostics ou d'interventions pratiquées par l'hôpital.

\section{Nous proposons de tenir compte de l'écart entre les nombres absolus de décès observés et de décès attendus.}

Nous proposons enfin de tenir compte des résultats des années précédentes pour évaluer la variabilité et de la tendance du nombre de décès d'une année à

Correspondance: l'autre.

Observatoire valaisan de la santé (OVS)

Avenue Grand-Champsec 86 CH-1950 Sion

Tél. 0276034961 julien.sansonnens[at]ovs.ch

\section{Conclusion}

La publication des indicateurs de mortalité de l'OFSP est réalisée dans l'intérêt des patients et des hôpi- taux, en ce qu'elle permet de mettre en évidence un certain nombre de situations potentiellement problématiques. L'accessibilité des résultats par le public, de même que le fait d'identifier par leur nom les établissements, constituent des pas importants vers plus de transparence dans le domaine des soins hospitaliers et sont des incitatifs à l'évaluation et l'amélioration de la qualité des soins. Néanmoins, ces indicateurs sont des indices d'un problème potentiel et ils doivent être interprétés correctement. Leurs limites doivent être bien comprises pour être un véritable outil d'amélioration de la qualité des soins dans les hôpitaux suisses. En complément au SMR, le calcul du nombre de décès attendus et observés aide à interpréter correctement ces indicateurs.

\section{Remerciements}

Nous adressons nos remerciements à Anthony Staines, Ph.D., chargé de programme "Sécurité des patients» à la FHV, pour ses commentaires critiques et constructifs.

\section{Références}

1 Donabedian A. The quality of care. How can it be assessed? JAMA. 1988;26o(12):1743-8.

2 Kohn LT, Corrigan JM, Donaldson MS, editors. To err is human: building a safer health system . Washington, DC: National Academy Press, Institute of Medicine; 1999.

3 Pineault R, Goulet L. Les indicateurs de santé: les utiliser, oui, mais ne pas en abuser. Ruptures. 1995;2:140-50.

4 Chiolero A, Paccaud F, Fornerod L. Comment faire de la surveillance sanitaire? L'exemple de l'Observatoire valaisan de la santé en Suisse. Santé Publique. 2014;26(1):75-84.

5 Lilford R, Pronovost P. Using hospital mortality rates to judge hospital performance: a bad idea that just won't go away. BMJ. 2010;340:c2016.

6 Luthi JC, Troillet N, Burnand B. Précautions et limitations lors de l'utilisation de la mortalité intra-hospitalière comme indicateur de la qualité des soins. Revue médicale de la Suisse Romande 2004;124:697-700.

7 OFSP. Indicateurs de qualité des hôpitaux suisses de soins aigus 2012. Berne;2014 Disponible en ligne sur: www.bag-anw.admin. ch/kuv/spitalstatistik/data/download/qip12_publikation. pdf?webgrab=ignore

8 OFSP. Indicateurs de qualité des hôpitaux suisses de soins aigus. Disponible en ligne sur: http://www.bag.admin.ch/ighs

9 Zaugg J. Classement des hôpitaux. Le droit de savoir. L'Hebdo. 2013;16:45-52.

10 Strupler P. A propos de l'article «A la recherche d'indicateurs de qualité pour les hôpitaux suisses». Prise de position du directeur de l'OFSP. Bull Méd Suisses. 2013;94(39):1384.

11 Carrel T. A la recherche d'indicateurs de qualité pour les hôpitaux suisses. Bull Méd Suisses. 2013;94(34):1270.

12 Dimick JB, Welch HG, Birkmeyer JD. Surgical Mortality as an Indicator of Hospital Quality. JAMA. 2004;292:847-51.
13 Gigerenzer G. What are natural frequencies? BMJ. 2011;343:d6386.

14 van Gestel YR, Lemmens VE, Lingsma HF, de Hingh IH, Rutten HJ, Coebergh JW. The hospital standardized mortality ratio fallacy: a narrative review. Med Care. 2012; 50(8):662-7. 